\title{
Freiheit in der Politischen Ökonomie von John Kenneth Galbraith*
}

Der jüngst verstorbene John Kenneth Galbraith ist einer der weltweit meistgeachteten Ökonomen. Seine intellektuelle Biografie reflektiert in einmaliger Weise die Sozial- und Wirtschaftsgeschichte des 20. Jahrhunderts. Durch eine kritische Methodologie sucht Galbraith die Wirtschaftstheorie vor ihrem Umschlag in Ökonomismus zu bewahren. In seinen politisch-ökonomischen Analysen findet er den Anschluss an die Sozialwissenschaften und an eine im Zeichen der Freiheit und Gerechtigkeit operierende praktische Philosophie. Indem er die gesellschaftliche Gestaltbarkeit der Ökonomie offenlegt und deren Wandel im Rahmen kultureller und politischer Vorgaben beschreibt, will Galbraith einer an der freiheitlichen Gestaltung ihrer sozioökonomischen Wirklichkeit interessierten Öffentlichkeit die konzeptionellen Mittel an die Hand geben, wirtschaftliche Macht zu identifizieren, zu kritisieren und zu modifizieren.

\section{Inhalt}

1. Einleitung

2. Galbraiths Kritik der ökonomischen Epistemologie und Methodologie $\quad 760$

3. Galbraiths Kritik der neoklassischen Gleichgewichtsannahmen 766

4. Galbraiths Kritik der neoliberalen Wirtschaftspolitik 772

$\begin{array}{ll}\text { 5. Zusammenfassung } & 779\end{array}$

Verzeichnis der verwendeten Siglen von John Kenneth Galbraiths Werk 780

$\begin{array}{ll}\text { Sekundärliteratur } & 780\end{array}$

\section{Einleitung}

Mit Milton Friedman und John Kenneth Galbraith verstarben unlängst die wirkmächtigsten amerikanischen Ökonomen des 20. Jahrhunderts. Obschon in der deutschen Wahrnehmung Friedman tiefer verankert ist, wird international vielfach der Titel des ,most widely known American economist of the twentieth century" seinem intellektuellen Gegenspieler Galbraith zugesprochen. ${ }^{1}$ Mit seinen theoretischen Schriften und sozialkritischen Romanen erwarb sich Galbraith von Russland über Indien bis Südamerika Weltruhm. Im öffentlichen Leben

* Für hilfreiche Kritik und Anregungen danke ich Silke Adam und Michael Pirson.

1 Parkers Biografie (2005) übertrifft die bislang vorliegenden Darstellungen zu Galbraith Leben und Werk an Genauigkeit und Detailtreue; ihr besonderer Vorzug ist die kompetente Einordnung der intellektuellen Biografie von Galbraith in den Gesamtzusammenhang der jüngeren Ökonomie- und Politikgeschichte. 


\section{Claus Dierksmeier}

der USA übernahm er die Rolle des streitbaren Keynesianers. In der akademischen Welt machte Galbraith sich durch betriebswirtschaftliche sowie ordnungspolitische Modifikationen der Keynesianischen Lehre einen Namen. Als politischer Vordenker, persönlicher Ratgeber und hochrangiger Diplomat hat Galbraith zudem auf die Entscheidungen mehrerer amerikanischer Präsidenten bedeutenden Einfluss genommen. In den letzten Jahren hat die Forschung zu Galbraith deutlichen Aufschwung erfahren (Laperce/Uzunidis 2006). Dabei stehen die ökonomischen Aspekte seines Wirkens im Vordergrund der Aufmerksamkeit. Bisher noch weniger berücksichtigt ist indes Galbraith eminenter Beitrag zur Sozial- und Politikphilosophie der Gegenwart.

In diesem Beitrag soll die freiheitsphilosophische Relevanz von Galbraiths Wirtschaftstheorie offengelegt werden. Diese resultiert aus der konsequenten Weise, in der Galbraith das ökonomische Denken in einen an individueller Freiheit und sozialer Gerechtigkeit zugleich interessierten Diskurszusammenhang einbindet und sich damit von den negativ-formalen Freiheitsbegriffen der angloamerikanischen Denktradition verabschiedet. Seinen positivmaterial ausgerichteten Freiheitsbegriff entwickelt Galbraith indes nicht aus philosophischen Prinzipienüberlegungen, sondern vermittels einer Analyse sozialwirtschaftlicher Sachzusammenhänge.

Im Folgenden wird zunächst Galbraiths Kritik an der vorherrschenden wirtschaftswissenschaftlichen Methodologie präsentiert. Seine Kernthese ist, dass die Ökonomik, die in der Ökonomie vorhandenen freiheitlichen Gestaltungsmöglichkeiten besser erfassen und nutzen könnte, wenn sie selbstreflexiver verführe. Sodann widmet sich der Artikel der Galbraith'schen Kritik der neoklassischen Wirtschaftstheorie. Hier geht es darum, die Gleichgewichtsannahmen der Neoklassik ${ }^{2}$ zu hinterfragen, um die auf ihnen beruhenden politischen Handlungsempfehlungen neoliberal-konservativer Natur zu kritisieren. Zuletzt folgt eine Untersuchung über den Zusammenhang von politischer Freiheit und Wirtschaftspolitik im Kontext der Globalisierung. Dabei argumentiert Galbraith zugunsten eines Begriffs wirtschaftlicher Freiheit, der sich affirmativ zu seinen kulturellen Voraussetzungen, seinen rechtlichen Ermöglichungsbedingungen und seinen sozialen wie umweltlichen Verpflichtungen verhält und so einer nachhaltigen Integration von persönlicher Autonomie und ökonomischer Wirklichkeit verpflichtet ist.

\section{Galbraiths Kritik der ökonomischen Epistemologie und Methodologie}

Vornehmstes Ziel der Wirtschaftswissenschaften ist für Galbraith die Emanzipation der Bürger zum autonomen Wirklichkeitsumgang. Um sich einer demokratischen Öffentlichkeit gegenüber verantworten zu können, muss Ökonomik als verständliche und selbstkritische Lehre von der Ökonomie betrieben werden. Zugangshindernisse zum Verständnis der Wirtschaft abzubauen, begreift er darum als die selbstverständliche Pflicht eines jeden Akademikers

2 Mit Galbraith wird hier unter Neoklassik die Wirtschaftstheorie in der durch Marshall (1890) repräsentierten Fassung verstanden. Der Begriff „,neoklassisch“ - geprägt von Thorstein Veblen - erklärt sich aus der Verschmelzung der „,klassischen“ Ökonomik (Smith, Ricardo) mit den nachträglich ausgearbeiteten Lehrsätzen der österreichischen Grenznutzenschule (Menger u. a.) zu einem einheitlichen Lehrgebäude. Näheres zum Begriff: Niehans/Jurg 1990: 159 ff. 
gegenüber der Öffentlichkeit. Doch genau diesem Anspruch, so Galbraith, genügen die Wirtschaftswissenschaften oftmals nicht. Statt unparteiisch wirtschaftliche Ereigniszusammenhänge zu untersuchen, resultiere die Theoriebildung innerhalb der ökonomischen Zunft nicht selten aus einem Neuaufguss längst überholter konventioneller Ansichten („,conventional wisdom"). Die Ökonomik verkünde Theorien, deren Bestandskraft nicht aus ihrer Erklärungsleistung, sondern vielmehr aus dem in sie eingegangenen ,,vested interest in painfully acquired error" resultiere (NIS 321) ${ }^{3}$. Wirtschaftswissenschaftler huldigten oftmals nicht der Wahrheit, sondern vielmehr, ,what is closest in belief and method to the scholarly tendency of the people who already have tenure in the subject." (AAL 135)

Im Bestreben, sich zu verwissenschaftlichen, haben sich die Wirtschaftswissenschaften im 18. und 19. Jahrhundert von ihrer Einbettung in moralphilosophische und sozialwissenschaftliche Kontexte gelöst. Die ehedem als Politische Ökonomie bzw. als Nationalökonomie mit konkreten ökonomischen Zuständen und deren rechtlichen Rahmenvorgaben befasste Wirtschaftswissenschaft hat sich immer mehr auf abstrakte Fragestellungen hin verengt. Das Ziel war, ökonomische Probleme sowohl wert- wie erfahrungsfrei zu bearbeiten und damit universal gültige Lösungen zu entwickeln. Damit aber schlug die Ökonomik einen bedenklichen Weg ein, meint Galbraith. Indem sie sich an einem formal-quantitativen, der Physik entlehnten Wissenschaftsideal orientiert, neigt die Wirtschaftswissenschaft nunmehr dazu, die Wissenschaftlichkeit von Theorien an der Quantifizierbarkeit ihrer Elemente und an der Mathematisierbarkeit ihrer Aussagen zu messen. ${ }^{4}$ Diese Orientierung steht aber einem unbefangenen Blick auf deskriptive Befunde wie normative Argumente entgegen. ${ }^{5}$ Gewisse Wirklichkeiten lassen sich nur qualitativ, nicht quantitativ beschreiben - und sind deswegen doch nicht weniger real; manche Argumente lassen sich erst spät und andere vielleicht niemals formalisieren - und sind allein darum doch nicht weniger vernünftig. Trotzdem wird in der Regel alles, was „oral rather than mathematical“ daherkommt, innerhalb der etablierten Ökonomik ,readily dismissed by the men of scientific reputation or pretension” (EPL 96). Und das führt zu einer bedenklichen Selbstimmunisierung gegenüber Information, die nicht zu den immer schon gehegten Überzeugungen passen will, was insbesondere gegenüber der Wahrnehmung von sozialen Veränderungen den Blick eintrübt: „Thus does a scientific or pseudoscientific posture direct economics away from accomodation to underlying social institutional change. And it does so with the blessing of presumptively scientific attitude, method and conscience." (EPL 96)

Dazu ein Beispiel: Das Ausgangsmodell der Neoklassik ist die Konkurrenzwirtschaft multipler kleiner Anbieter, wie sie für die Zeit von 1750-1850 typisch war, und nicht die gegenwärtige Realität oligopoler Großunternehmen mit Einfluss auf die makroökonomischen

3 Die Zitationen folgen dem im Anhang abgedruckten Siglenverzeichnis.

4 Vgl. Shackle 1972: 360; McCloskey 1994: 9 ff; Colander/Klamer 1987: 95-111; Summers 1991: 129-148; Solow 1997: 39-58.

5 Vgl. Bergmann 1989: 29-37, bes. 33. Siehe auch bereits Chamberlain 1948: 95-108; sowie Sen 1999: 139-146 zu den Vorteilen qualitativer (statt quantativer) Beschreibungstechnik bei Galbraith. Siehe auch Reich 1999: 88-100: „A theory grounded in rational self-interest may explain much that people do, but it offers only limited guidance for determining what society should try to accomplish." (96); Roll 1989: 113-122. 


\section{Claus Dierksmeier}

Rahmenbedingungen. So wurde etwa der Druck, den Gewerkschaften und Großkonzerne (durch Absprachen über Lohnzahlungen) auf die gesellschaftliche Preisentwicklung ausüben, lange Jahre nicht studiert bzw. als theoretisch irrelevant abgetan. Statt an den Strukturen der modernen Industriegesellschaft orientierte sich die akademische Preistheorie bis weit in die 70er-Jahre hinein lieber an kleinen agrarischen Familienbetrieben, die aufgrund ihrer winzigen Betriebsgröße keinerlei Möglichkeiten zur Verzerrung der (von der Neoklassik postulierten) Gleichgewichtspreise am Arbeitsmarkt ausüben können (NIS 411). So aber begab sich die Ökonomik der Möglichkeit, die tatsächlichen Lohn- und Preisentwicklungen angemessen zu beschreiben bzw. - wie die Stagflationskrisen der 70er-Jahre demonstrierten - für eben diese Verhältnisse die richtigen wirtschaftspolitischen Empfehlungen zu formulieren.

Die darin zutage tretende Abneigung, historischen Wandel aufzuarbeiten, ist zum Teil dem naturwissenschaftlichen Erkenntnisideal der Ökonomik geschuldet. Der Gegenstand der Physik bleibt sich gleich; Wissenschaftsfortschritt resultiert mithin aus einer immer genaueren und immer weiter spezialisierten Beschreibung dessen, was von jeher der Fall ist. Dem nacheifernd hat sich die akademische Ökonomik entsprechend eine bessere (quantitative) Erfassung ihres Gegenstandes erhofft und durch eine immer weiter fortschreitende Spezialisierung und eine immer feinere Untergliederung in Teildisziplinen zu erzwingen gesucht (NIS 411). Im Ergebnis hat dies indes eher eine Abschottung der ökonomischen Spezialbereiche gegen wichtige (qualitative) Information aus umliegenden Disziplinen sowohl innerhalb wie außerhalb der Wirtschaftswissenschaften bewirkt und damit zu einem bedenklichen Erkenntnis- und Realitätsverlust seitens der Ökonomen geführt.

Es gibt ja nirgends „die Wirtschaft“ als überzeitlich ein für allemal festliegende Entität, sondern allerorts tritt wirtschaftliches Handeln in unterschiedlicher Form auf und unterliegt mannigfaltigen, oft simultan auftretenden kulturellen wie politischen Einflüssen. Ökonomische Ereignisabläufe sind zudem hochgradig wechsel- und rückbezüglich. Von daher entpuppt sich eine Abstraktion von sowohl eigensystemischen wie umweltlichen Einflüssen als zwangsläufige Fehlerquelle (NIS 403). Wenn zum Beispiel gesellschaftlicher Wandel in mehreren ökonomischen Subsystemen gleichzeitig auftritt, muss die Anwendung von ceteris paribus-Regeln einer umfassenderen Perspektive, die möglichst alle im Wandel begriffenen Entitäten einbezieht, weichen. Tut sie das nicht, blendet sie tatsächlich kausale Faktoren aus und sieht sich in Ermangelung derselben gezwungen, an und für sich irrelevanten Phänomenen eben diese Kausalität zuzusprechen. Daraus resultieren dann doppelt verfehlte Handlungsempfehlungen: Zunächst wird zur Einwirkung auf Faktoren geraten, die gar nicht im erhofften Sinne ereigniskausal sind; anschließend wird aus dem Scheitern solcher Maßnahmen geschlossen, dass noch nicht stark genug eingegriffen wurde; der Mitteleinsatz wird erhöht, es kommt zu sich dramatisch aufstufenden Fehlschlägen (NIS 405).

Ein klassisches Beispiel hierfür ist die wirtschaftstheoretische Einschätzung des Marktes: Ein Markt, auf dem unter fairen Konkurrenzbedingungen gehandelt wird, fällt nicht vom Himmel, sondern ist Resultat oft jahrhundertelanger kultureller wie rechtspolitischer Bemühungen, individuelles wie organisationelles free-rider-Verhalten auszumerzen. ${ }^{6}$ Eine Theorie, welche jedoch aus methodischen Gründen alle soziokulturellen Rahmenvorgaben ausblendet, muss den Markt indes gleichsam als ein sich selbst ins Gleichgewicht bringendes Natur-

Sahlins 1972: 85 f.; Lowry 1987; Menninger 1969: 212 f. 
phänomen konzipieren. Die daraus resultierende Handlungsempfehlung ist ein politisches Gestaltungsverbot; man wird mithin gestörtes Marktgeschehen durch Deregulierung zu optimieren suchen. Bei ausbleibendem Effekt wird gefolgert, der Markt sei noch nicht genügend dereguliert worden; und das im Zweifel solange, bis der anfangs noch partiell intakte Markt vollends zusammenbricht. Die Schuld wird sodann Macht- und Informationsasymmetrien (z. B. oligopolen Anbietern) in die Schuhe geschoben, deren verhängnisvolles Wirken indes zuallererst durch eben jene Deregulierung ermöglicht wurde. Wer hingegen die multikausalen Rahmenbedingungen von Märkten in seine Analyse mit aufnimmt, wird umgekehrt durch ein politisches Gestaltungsgebot dem Wunsch nach fairen Austauschbeziehungen zu entsprechen suchen.

So handelt es sich bei der mathematischen Engführung der ökonomischen Theorie also keineswegs um eine unschuldige akademische Abstraktionsleistung: Wann immer nämlich außerwirtschaftlichen bzw. nicht-quantifizierbaren Faktoren wirtschaftsprägende Kraft zukommt, verleitet die Ausblendung derartiger Aspekte die Ökonomie zu Fehlschlüssen. Eine Ökonomik, die etwa den Bürger durch ihre methodologischen Festlegungen daran hindert zu verstehen, wie und von wem er in seinem Wahlverhalten am Markt beeinflusst wird (Werbung, Marketing, Tiefenpsychologie), dient nicht dem Allgemeininteresse, sondern identifizierbaren, so jedoch unidentifiziert bleibenden Spezialinteressen. Ähnlich lässt die in den Wirtschaftswissenschaften übliche Verbannung des Themas der „Macht“ den Einfluss von organisationeller Macht auf ökonomische Regelungs- und Verteilungsfragen zwar aus der Theorie, nicht aber aus der Praxis verschwinden (AL 136). Wo immer dieser Einfluss sich bemerkbar macht, stellt folglich die Theorie die Wirklichkeit nicht dar, sondern verschleiert sie. Ökonomik wird somit - wie Galbraith das spitz formuliert - zum ,influential and invaluable ally of those whose exercise of power depends on an acquiescent public." (AAL 151)

Jener Einfluss der Ökonomik auf Ökonomie und Gesellschaft wird verstärkt durch die Weigerung der Ökonomen, überhaupt anzuerkennen, dass ihr Forschungsgegenstand im Rahmen begrifflicher Konstruktion von ihnen selbst definiert wird. ${ }^{7}$ Die Gefahr ist also, dass der Ökonomik von ihren Vertretern und sodann auch vonseiten der Gesellschaft zu wenig Skepsis entgegengebracht wird, weil ihre Empfehlungen als „wissenschaftlich“ bzw. überparteilich hingenommen werden. Im Hinblick auf die soziale Verantwortung der Wirtschaftswissenschaftler ist das misslich: Je weniger sich nämlich die Ökonomik als wirklichkeitsgestaltend wahrnimmt, um so unbefangener - d. h. gleichgültiger gegenüber den sozialen Konsequenzen ihrer Ratschläge - wird sie verfahren: sie hat die wirtschaftlichen „Gesetze“ ja nur verkündet, nicht gemacht. Da aber wirtschaftspolitische Handlungsempfehlungen sich notwendig auf wirtschaftstheoretische Beschreibungen stützen, gestaltet die Ökonomik de facto - aller methodologisch postulierten Neutralität zum Trotz - die von ihr analysierte Wirklichkeit mit. Galbraith will deshalb die Ökonomik als Sozialwissenschaft rekonzipieren, um so einen sowohl selbstkritischen wie demokratisch verantwortbaren Gebrauch jener Geistesmacht zu ermöglichen (Boulding 1969: 1-12).

7 Mit dieser Ansicht steht Galbraith nicht alleine: „In natural science, what is thought is built upon what is seen; but in economics, what is seen is built upon what is thought." (Shackle 1972: 66); zu dieser These ausführlicher Samuels 1984: 67-76. 


\section{Claus Dierksmeier}

Viele Ökonomen halten dem entgegen, man wolle - und könne - doch nicht Wertwissenschaft betreiben. Im Gefolge des Werturteilsstreits (Backhaus/Hansen 2000) hat sich die Mehrheit der Ökonomen der Ansicht angeschlossen, die Wirtschaftswissenschaften hätten nicht über die Ziele des Wirtschaftens zu befinden, sondern dienten allein einer Aufklärung über den bei gesetzten Zwecken intelligentesten Gebrauch vielfach verwendbarer, knapper Mittel. ${ }^{8}$ Bis heute wird diese Verweigerung eines Diskurses über die angemessenen (individuellen wie gesellschaftlichen) Zwecke ökonomischen Handelns legitimiert mit einer bescheidenen Abstinenz von Letztbegründungen und/oder einem schneidigen Verweis auf die nicht quantifizierbare Natur der in Zielfestlegungen unweigerlich zum Zuge kommenden qualitativen Wertungen.

Galbraith zufolge befindet sich die Wirtschaftswissenschaft hier in einer gefährlichen Selbsttäuschung. Denn wenn ihr explizite (kategorische) Orientierungen fehlen, so muss sich die Ökonomik doch wenigstens implizit (hypothetisch) ausrichten, um auch nur einfachste strategische Empfehlungen formulieren sowie eine ihre Teildisziplinen integrierende Forschungsperspektive gewinnen zu können. Darum operieren die Wirtschaftswissenschaften mit einem auf den ersten, aber nicht mehr auf den zweiten Blick unschuldig erscheinenden Notbehelf: nämlich generell ein „Mehr“ (in Fragen der Güterversorgung, Produktion, Beschäftigung etc.) gegenüber einem ,Weniger“" vorzuziehen. Nicht zufällig also wird wirtschaftlicher Erfolg anhand des Bruttoinlandprodukts evaluiert (NIS 408). Im Schutze der Behauptung, die Ökonomik stelle lediglich sicher, dass der Gesellschaft hinreichende Mittel zu den von ihr verfolgten Zwecken zur Verfügung stünden, avanciert das Produktionswachstum unbesehen zum normativen Ziel aller ökonomischen Theorie und Praxis. Aus der Arbeitshypothese wird flugs ein die Wirtschaftpolitik gestaltender Imperativ.

Die Prämisse, dass menschlichen Zwecken samt und sonders eher durch ein „Mehr“ als durch ein „Weniger“ an Produktion gedient sei, versteht sich allerdings durchaus nicht von selbst. Weil und insofern sie der Maxime der maximierten Ressourcennutzung entgegenstehen, gehen nämlich Sinn und Bedeutung unverzweckter natürlicher wie kultureller Räume in dieses Wertkalkül nicht ein. Sofern die Gesellschaft sich nicht gegen den Appell zum unbegrenzten Wirtschaftswachstum zur Wehr setzt, liefert sie jedoch die Gestaltung des öffentlichen Raumes an den Wirtschaftswissenschaftler als „highest arbiter of social policy“ aus (NIS 408). Er wird alle gesellschaftspolitischen Optionen einer cost/benefit-Analyse unterziehen und von jeglichem abraten, was mehr kostet als einbringt. Dass allerdings dabei in die ökonometrische Bewertung nur solche Aspekte einfließen, die sich quantitativ messen lassen, bringt derartige Entscheidungsfindungsprozesse in eine materialistische Schieflage. Ideelle Gebilde lassen sich zumeist weit schlechter taxonomieren als körperliche Dinge. Sie finden in ökonomischen Kalkülen weniger Berücksichtigung. Die Fokussierung auf mehr und mehr Produktion und folglich, um diese abzusetzen, auf immer extensiveren und intensiveren Konsum drängt alternative Lebensentwürfe zusehends zurück: Ziele wie „more sharing of work“, „more relaxed conditions of toil or less air and water pollution“, ,,a larger amount of

8 Etwa in der zum Klassiker avancierten Formulierung von Lionel Robbins: „Economics is the science which studies human behavior as a relationship between ends and scarce means which have alternative uses" (1932: 16). Siehe auch Friedman 1953: 3-43. 
leisure“, etc., die unter anderem auf eine Drosselung der Produktionszuwächse angewiesen wären (NIS 408), fallen dieser Festlegung quasi-automatisch zum Opfer.

In der Optik des Bruttoinlandsprodukts kann ein Zuwachs an individueller Muße nicht gemessen werden; wohl aber ein Zuwachs an Gütern in Privatbesitz. So wird etwa der private Kauf und Betrieb eines Autos gesellschaftlichem Wohlfahrtsgewinn gleichgesetzt. Auch und gerade eine zu hohe Dichte an Privatfahrzeugen führt durch die anfallenden Regelungsaufgaben (Verwaltung, Verkehrssicherheit), die nötige Infrastruktur (Straßenbau, Regelungstechnik), den erhöhten Rohstoffverbrauch und anfallende Schadensbeseitigungsmaßnahmen (Unfallkosten, Umweltschäden) zu einem erhöhten Bruttoinlandsprodukt (EPL 100). Eine Gesellschaft, in der jeder Einwohner über 10 Autos verfügte, würde nun zwar ein sehr hohes Bruttoinlandsprodukt aufweisen, ob deren tatsächliches Wohlfahrtsniveau aber das einer Gesellschaft mit geringerer Fahrzeugdichte überträfe, ist fraglich. In letzter Hinsicht fallen die Lasten des Automobilverkehrs ja entweder - wie typischerweise in Europa - auf dem Umweg über Steuern und Abgaben oder aber - die amerikanische Variante - in Form von unbehobenen öffentlichen Ärgernissen (veraltete Regelungstechnik, mangelnde Integration des Fuß- und Radverkehrs, Staus), wiederum auf das Individuum zurück. Von der Umweltvernutzung und -verschmutzung ganz zu schweigen.

Die Abstraktion der Ökonomie von ihrer humanen Zielbestimmung führt so oftmals zu einem ,progress toward the wrong goals“ (NIS 409). Demgegenüber plädiert Galbraith, dass sich eine Gesellschaft ihre (moralischen, ästhetischen, politischen) Werte durchaus ökonomischen Wohlstand kosten lassen solle (EPL 104). Zwar werden in armen Gesellschaften die im engeren Sinne ökonomischen Ziele auch die politisch vordringlichen sein; jede Gesellschaft hat schließlich ihren Mitgliedern die Lebensgrundlagen zu sichern. Je wohlhabender eine Gesellschaft aber ist, desto weniger sinnvoll ist es, Politik und Kultur dem Imperativ eines ungebremsten ökonomischen Wachstums zu unterwerfen. Zusehends mehr müssen Fragen der Lebensqualität Vorrang vor rein quantitativen Zwecksetzungen erhalten (EPL 98), denn letztlich ist der Mensch der Zweck der Wirtschaft - und nicht umgekehrt (EPL 101). Deshalb schlägt Galbraith (in der Nachfolge von John Stuart Mill) mit der Deckung des Grundgüterbedarfs eine Umkehr der Blickrichtung vor. Er plädiert dafür, die Wirtschaftswissenschaft Zug um Zug mit dem Steigen des allgemeinen Wohlstandsniveaus aus der Verantwortung für gesellschaftliche Zielsetzungen herauszudrängen (NIS 407) bzw. (wie er seit den Achtzigern favorisiert) ihrerseits für den gesellschaftlichen Diskurs um das gute Leben zu öffnen. Innerhalb einer solchen wert- und umweltbezogenen Ökonomik wären dann Perspektiven für wirtschaftliches Handeln zu erarbeiten, an dem dieses qualitativ evaluiert werden könnte.

Dem steht seitens der ökonomischen Zunft einiger Widerstand entgegen. Mit einer Depotenzierung der ökonomischen Werte im Rahmen der gesamtgesellschaftlichen Prioritätensetzung geht zwangsläufig ein sozialer Bedeutungsverlust des fachökonomischen Wissens und damit eine Entmachtung seiner Gralshüter einher. Die Ökonomenzunft hat aber naturgemäß kein Interesse daran, obsolet zu werden (EPL 93) und hält von daher am Fortbestand des Wachstumsparadigmas fest (EPL 95). Dieser Trend wird durch die zunehmende Privatisierung des akademischen Sektors nicht unerheblich verstärkt. Etliche Ökonomen erhalten heute ihr Brot - oder doch wenigstens ein sehr reichhaltiges Zubrot - aus der Privatwirt- 


\section{Claus Dierksmeier}

schaft (NIS 373). Neben der in allen Wissenschaften zu beobachtenden Tendenz, dem akzeptierten Wissen den Vorzug vor unbequemen Neuerungen zu geben, werden die Wirtschaftswissenschaftler also zudem noch durch die Neigung, nicht die Hand zu beißen, von der man gefüttert wird, vom Aussprechen unbequemer Wahrheiten zurückgehalten (AP 130).

Die größte Chance für eine Abkehr von der Vergötterung des Produktionszuwachses liegt darum in einem allseits bemerkten Scheitern des Paradigmas. Insofern die Ökonomik letzten Endes an der Praktikabilität ihrer Handlungsempfehlungen gemessen wird, kann sie ein bestimmtes $\mathrm{Ma} ß$ an Realitätsferne und Steuerungsversagen nicht unbeschadet überstehen. Wenn der „march of events“ die „,conventional wisdom“ des Faches so nachdrücklich Lügen straft, dass Aussicht besteht, mit dem Ruf nach Paradigmenwechsel akademische Karriere zu machen, werden sich zusehends mehr Ökonomen dafür erwärmen, die beschriebene Realität näher der beobachtbaren Realität anzupassen. Und mit der Ablösung des Entrepeneurkapitalismus durch managementgeleitete Großunternehmen, meint Galbraith, wurde ein eben solcher „march of events“ eingeleitet, der die Ablösung des neoklassischen Forschungsparadigmas erzwingt.

\section{Galbraiths Kritik der neoklassischen Gleichgewichtsannahmen}

Der Kunde ist König! - so lautet die Fundamentalthese jener Ökonomen, die glauben, dass die marktwirtschaftlichen Handlungsabläufe ,von unten“, d. h. von der freien Wahl des Konsumenten, und mithin quasi-demokratisch gesteuert werden (NIS 210). Die philosophischen Grundlagen jener Ansicht stammen von Jeremy Bentham, ihre ökonomische Ausarbeitung beginnt unter David Ricardo, kanonische Gestalt erfährt sie bei Alfred Marshall. Im Kern geht es darum, die althergebrachte philosophische Debatte um das objektiv Gute ein für allemal zu verabschieden. Gut - so der britische Utilitarismus unisono mit der ein Jahrhundert später kommenden österreichischen Grenznutzenschule - ist, was ein jeweiliges Subjekt dafür hält; Ende der Debatte. Dieser Ansatz resultiert in folgender Politiktheorie:

Da niemand so gut wie das betroffene Subjekt selbst von dessen Vorstellungen vom Guten Kenntnis hat, wird jede von außen kommende Verhaltenssteuerung notwendigerweise suboptimale Ergebnisse liefern. Das Gemeinwohl wird dabei mit der Summe des Wohlseins aller Einzelnen gleichgesetzt. Es steigt proportional zum Zuwachs an individuellen Wahlmöglichkeiten. Alle Beschränkungen derselben sind daher prinzipiell dem Gemeinwohl abträglich und nur legitimiert, wenn sie dem Subjekt als rational sinnvoller Umweg zum eigenen Wohlbefinden erklärt werden können - wie zum Beispiel rechtliche Koordinationsregeln, die, obschon lästig, Konflikte vermeiden helfen. Die beste Politik ist demnach diejenige, welche das denkbare Minimum an individuellen Willkürbeschränkungen erreicht. Um die politischen Gestalter dahingehend dirigieren zu können, sollen die Individuen durch ihre Stimmabgaben den Souverän spielen (Waligorski 1990).

Parallel zu diesem Politikverständnis formuliert die moderne Ökonomik ihre Theorie der „consumer sovereignity“, derzufolge die Wirtschaft vom Konsumenten durch die Abgabe seiner Kaufentscheidung gelenkt wird (NIS 211). Seinem individuellen Nutzen nachstrebend kauft der Einzelne, was ihm (relativ am meisten) gefällt, und informiert damit den Markt, welche Bedürfnisse (relativ am dringlichsten) zu erfüllen sind. Nur der Konsument selbst hat 
eine intime Kenntnis des Katalogs seiner Bedürfnisse; er allein wird darum seine Geldmittel optimal einsetzen und zwar stets so, dass die Grenznutzen aller von ihm erworbenen Güter einander gleichen: Man wird also z. B. nicht so unverständig sein, noch eine zusätzliche Einheit Salz zu kaufen, wenn stattdessen ein Anstieg des heimischen Zuckervorrats zu höherem Eigennutz verhilft. Jede Einmischung in die individuelle Kaufentscheidung vonseiten der Regierung muss jenes grenznutzentheoretische Kalkül verzerren und wird insofern per definitionem die Ressourcen des Individuums weniger effizient verteilen, mithin deren Glück und folglich die als die Summe aller individuellen Befriedigungsmomente definierte Wohlfahrt der Gesellschaft mindern (NIS 213).

Folgen wir der neoklassischen Theorie noch einige Schritte weiter: Die Unternehmen werden durch die Abgabe der in den Preisen kommunizierten Information geradezu ,gezwungen“", sich an die Erfüllung der gesellschaftlich vorhandenen Bedürfnisse zu machen. Denn die Anbieter stehen miteinander in scharfer Konkurrenz. Um am Markt zu bestehen, erstreben sie die ständige qualitative Verbesserung ihres Angebotes und dessen quantitative Vermehrung. Um technisch effizienter zu produzieren, reinvestieren die Unternehmer ihre Profite. Und da die Konkurrenz mitzieht, gilt es die Profite zu maximieren, um so beim Wettlauf um die beste Produktionstechnik vorne zu liegen. Im Gefolge dessen sinken die Stückkosten stetig und diese Ersparnis wird (wiederum aufgrund von Anbieterkonkurrenz) an den Konsumenten in Form sinkender Preise weitergegeben. Im Endeffekt wird am Markt zum jeweils günstigsten Preis genau das feilgeboten, was die Konsumenten wünschen; jeder erlangt also das theoretisch mögliche Maximum an Nutzen: Der Kapitalismus schafft die beste aller möglichen Welten.

Das hier skizzierte Modell funktioniert recht gut, wenn man sich als Unternehmer einen Manufakturbesitzer im 19. Jahrhundert denkt, der mit Eigenkapitalanlage unter Bedingungen nahezu vollständiger Marktkonkurrenz operiert: Durch ein Zusammenwirken von Marktkonkurrenz einerseits und Geldpolitik andererseits können derartig operierende Unternehmer in der Tat zu einer wohlfahrtsdienlichen Produktionsweise angetrieben werden. Das vielzitierte „Saysche Gesetz“ (Say 1803: 138 f.) beruht beispielsweise auf dem Postulat von Unternehmerzwang durch Marktkonkurrenz: Wenn ein Gut überproduziert wird, bleibt dem Produzenten nichts anderes zu tun übrig, als dessen Preis immer weiter zu senken, bis es wieder Abnehmer findet. Der fallende Preis kommuniziert anderen Produzenten, dass sich ein Investment in dieses Gut nicht lohnt. Überproduktion wird abgebaut; also herrscht alsbald ein erneutes Gleichgewicht aus Angebot und Nachfrage (NIS 220). Und ein Lehrbuchbeispiel für die geldpolitische Steuerung des unternehmerischen Handelns ist, wenn der Staat zur Abkühlung einer überhitzten Spekulation künstlich die Geldmenge verknappt. Die Unternehmer bekommen am Geldmarkt aufgrund angehobener Zinssätze Investivkapital nur noch gegen erhöhte Preise; etliche zuvor noch marginal profitable Investitionen rentieren sich fortan nicht mehr und unterbleiben. Der Markt kühlt sich ab.

Galbraith bestreitet nicht, dass auch heute noch Märkte existieren, die dem hier skizzierten Modell nahekommen: der kommunale Gemüsemarkt lokaler Anbieter etwa. Er behauptet aber, dass im Rahmen industrieller Produktion - mithin angesichts weit über der Hälfte des Wirtschaftsvolumens westlicher Gesellschaften - durchaus nicht jene ,,accepted sequence“ einer „Wirtschaftssteuerung von unten“, sondern eine „revised sequence“ zu 


\section{Claus Dierksmeier}

beobachten ist, derzufolge die wesentliche ökonomische Lenkungskraft nicht bei Konsumenten, sondern bei den Unternehmen liegt (NIS 212 ff.). Nicht mehr die isolierte Wahlfreiheit der Abnehmer, sondern die organisierte Macht der Anbieter gibt den Takt vor. Die Gründe dafür sind praktischer Natur: Durch fortlaufende Modernisierung haben sich die industrielle Güterproduktion und ihr Absatz in den vergangenen Jahrzehnten radikal verändert. Ein vermehrter Einsatz von Technologie hat zu einem stark gestiegenen Bedarf an Kapital und qualifiziertem Personal geführt; zugleich haben sich Produktionsvorlaufzeiten und Planungsbedarf erheblich erhöht. Ein Flugzeugträger etwa wird mit einem ungleich höheren technischen, personellen und finanziellen Aufwand produziert als eine Pferdekutsche. Das zieht wichtige betriebs- und volkswirtschaftliche Konsequenzen nach sich (AP 141).

Statt beim privaten Kapitalgeber, der als Unternehmenseigner, Erfinder und Entscheider zugleich wirkte, liegt heute die finanzielle Last bei den Anteilseignern, die technische Konstruktion bei einem Stab von Spezialisten und die personelle Unternehmensführung beim Management. Die Entscheider im modernen Großunternehmen unterliegen weit weniger Zwängen als ehedem der Privatkapitalist. Sie haften finanziell nicht oder nur geringfügig für das Unternehmen und das verändert ihre Einstellung zu Profitmaximierung und Unternehmensführung gewaltig (EPP 266). Typischerweise kann das Management ungestraft von dem neoklassischen Postulat abweichen, sein Einkommen am Unternehmensprofit auszurichten, und stattdessen Höhe und Zusammensetzung desselben diskret für sich selbst verabschieden (EIF 27). Das hilft, das allseits diskutierte Phänomen zu erklären, dass CEO-Gehälter in aller Regel auch dann noch steigen, wenn Unternehmen schlechte Bilanzen vorlegen (CC 74). Die Anteilseigner, denen rechtlich gesehen ja das Unternehmen gehört, sind demgegenüber weitgehend machtlos. Selbst wenn sie lediglich an der Maximierung ihrer Dividenden und von daher an einer strikten Entlohnung des Managements gemäß seinem Beitrag zum marginalen Produktivitätszuwachs interessiert wären, wie es ihnen die neoklassische Theorie - dem Zuwachs an moralisch motivierten Anlegerverbänden sowie der Nachfrage nach ,ethischen“ Investmentfonds augenfällig zuwider - andichtet (EIF 31), es hilft ihnen nichts: mangels geeigneter corporate governance-Strukturen. ${ }^{9}$

Was sich hinter diesen technischen Überlegungen verbirgt, ist nichts weniger als eine Vindikation des Begriffs unternehmerischer Freiheit. Denn wenn, anders als von der Neoklassik angenommen, die Manager nicht durch die Gehaltsschraube zu ganz bestimmtem Handeln genötigt werden, woran richten sie dann ihre unternehmerische Tätigkeit aus? Außerhalb mathematischer Idealwelten gestattet die Maßgabe zur Profitmaximierung keine eindeutigen Handlungsableitungen (Marglin 1999: 114-138, 134). Unter real existierenden Unsicherheitsbedingungen müssen also andere Maximen her, um das Management zu orientieren; wie z. B. das Streben des Managements, korporative sowie gesellschaftliche Rollenerwartungen zu erfüllen (identification) und die Unternehmensorganisation Eigeninteressen anzupassen (adaptation). ${ }^{10}$ Die neoklassische Theorie hat sich solcher Phänomene lange Zeit nicht angenommen; einmal sind solche Maximen ,not easily adapted to the simplifications of mathematics and symbolic logic" (NIS 146), zum anderen machen sie das Argument zuschanden, Unternehmen könnten allein schon deshalb keine moralische Verantwortung für

9 Vgl. Cyert/March 1992; Marris 1964; Unseem 1996.

10 Vgl. Williamson 1970; Wood 1975, Galbraith 1984: 43-60; Leibenstein 1987. 


\section{John Kenneth Galbraiths Politische Ökonomie}

ihr Handeln übernehmen, weil ihnen (durch Markt und Anteilseigner bedrängt) dazu gänzlich die Freiheit fehle. Anzuerkennen indes, dass das Management sehr wohl in der Lage ist, sich den nötigen Raum zu verschaffen, um systemische Eigeninteressen zu verfolgen, heißt nicht nur das neoklassische Dogma infrage zu stellen, dass private Unternehmen ihre Konsumenten jederzeit zu einem Minimum gesellschaftlicher Kosten mit einem Optimum an Qualität versorgen. Es heißt auch zuzugeben: Wo Freiheit zum egoistischen Abweichen von der Maximierungslogik besteht, gibt es auch eine Freiheit zum alterozentrierten Handeln. Und aus jener Freiheit resultiert die Verpflichtung des Managements zu ihrem verantwortlichen Gebrauch.

Die corporate bureaucracy in modernen Großunternehmen ist an der Stabilität ihrer Jobs und deshalb an der Akzeptanz ihres Handelns im organisationellen wie gesellschaftlichen Kontext interessiert. Die Unternehmenspolitik eines aufgeklärt handelnden Managements wird von daher nicht immer Profite maximieren, meint Galbraith. ${ }^{11}$ Denn radikale Profitverfolgung bringt das Unternehmen dreifach in die Bredouille: Zum einen kann sie einen existenzgefährdenden Verdrängungspreiskampf mit der Konkurrenz einleiten; zum anderen führt eine allzu aggressive Lohnpolitik (zur Kosteneinsparung) zum Konflikt mit der (organisierten und streitbaren) Arbeitnehmerschaft; und wenn drittens das Unternehmen allzu rabiat Stakeholder-Interessen zugunsten der Bilanz aufopfert, bringt es schließlich den Gesetzgeber und die Gesellschaft insgesamt gegen sich auf. All das gefährdet die langfristige Geschäftstätigkeit und damit die berufliche Sicherheit der Manager. Wenn das Unternehmen indes einer moderateren Gewinntätigkeit nachgeht, kann es wachsen, ohne notwendigerweise zu Verdrängungswettbewerb, Straßenschlachten und einem Kulturkampf mit der Gesellschaft Anlass zu geben. Dabei sichert der schiere Größenzuwachs die berufliche Position des Managers, und je mehr Personal er unter sich hat, desto mehr Gehalt wird er beziehen. Die unternehmerische Freiheit des modernen Managements findet also nicht - wie von der Neoklassik postuliert - in der Profitmaxmierung ihre Grenze, wohl aber - wie Galbraith gesehen hat - im stetigen Anwachsen des Produktionsvolumens ihr Ziel.

Der ökonomische Strukturwandel komplettiert das Bild: Die Größe der modernen Industriekomplexe und der technische Aufwand ihrer Produktion setzt sie erheblichen Risiken durch Geldpreisschwankungen am Kreditmarkt aus. Um dieses Risiko zu verringern, findet sich in modernen Großunternehmen eine starke Neigung zur Bildung von - mit dem Dogma strenger Profitmaximierung ebenfalls nicht verträglichen - Rückstellungen, die das Unternehmen von externen Kapitalzuflüssen unabhängig machen sollen. Je größer das Unternehmen, desto leichter lassen sich Rücklagen bilden und in Krisenzeiten als Quersubventionen verwenden. Die systemische Perspektive des Managements ist dabei, ganz rational, viel langfristiger ausgerichtet als die eines Privatunternehmers, der ja nach ausreichender Gewinnmitnahme seinen Geschäftsbetrieb in schlechten Zeiten einfach aufgeben kann. Das Management als Lohnempfänger ist und bleibt dagegen von einer dauerhaften Geschäftstätigkeit abhängig.

11 Diese These hat bei ihrer Publikation zunächst heftigen Widerspruch ausgelöst. Vgl. Solow 1967: 100-108; Marris 1968: 37-45; Demsetz 1970: 481-484. Sie wurde aber schon wenig später empirisch und analytisch unterstützt: vgl. Caves 1970: 283-302; Williamson 1970: 93 ff.; Iwand/Thomassen 1979: 331-351. 


\section{Claus Dierksmeier}

Ein langfristig ausgerichteter Geschäftsbetrieb braucht eine stabile Nachfrage. Nun mag Says Gesetz, dass bei sinkenden Preisen jede Ware letztlich ihren Abnehmer findet, zwar im Bezug auf knappe Bedarfsgüter gelten. Heute allerdings findet der überwiegende Handel in Waren statt, die der Käufer durchaus nicht zum Überleben braucht (wie Prestigegüter, Markenartikel und Unterhaltungselektronik). Die subjektive Meinung, die der Käufer über sein Vermögen und über die Welt im Allgemeinen hegt, beeinflusst die Nachfrage solcher Güter und macht so einen Teil ihres objektiven Verkehrswertes aus. Man kauft oftmals auch - und bisweilen gerade dann - nicht, wenn Preise sinken (Liquiditätspräferenz). Jedoch können sich moderne Unternehmen ungleich schwerer als ihre frühkapitalistischen Vorläufer auf Schwankungen der Nachfrage einstellen. Anders als in der präindustriellen Ära lassen sich Nachfrageschwankungen bei planungs- und kapitalintensiven Industriegütern (wie z. B. Autos) weder durch drastische Änderungen der Menge oder Art der Produktion noch über radikale Preissenkungen abfangen. Sowohl von Konsumenten- wie von Unternehmerseite aus wird also dem von Say postulierten Marktgehorsam getrotzt. Der darauf gestützten neoklassischen Forderung nach einer volkswirtschaftlichen Enthaltsamkeit des Staates ist mithin die betriebswirtschaftliche Grundlage entzogen.

Wenn Unternehmen eher auf eine Stabilisierung der Nachfrage denn auf maximale Profite setzen, gilt es, auf die individuelle wie gesamtgesellschaftliche Abnahme und die sie stützenden Einstellungen Einfluss zu nehmen. Es liegt im systemischen Interesse der Unternehmen, die „freie“ Kaufentscheidung des Einzelnen zu manipulieren und somit die von der Neoklassik aufs Podest gehobene „consumer sovereignity“ zu untergraben. Werbung gäbe es nicht, wäre sie erfolglos. Deshalb muss, wer angesichts der quantitativen Dominanz und qualitativen Penetranz derselben die These von der „,consumer sovereignity“ aufrechterhalten will, konsequentermaßen behaupten, dass Werbung lediglich informativ wirke $-\mathrm{d}$. h. die Rationalität (im Nutzenkalkül) der Kaufentscheidung durch produktrelevante Information erhöhe. Indes wird Werbung suggestiv betrieben und appelliert gezielt an irrationale Motivationskräfte. Gestützt auf akademisch-psychologische Beratung nimmt sie Lebensängste und Sehnsüchte in den Dienst, um vorhandene Bedürfnisse zu verstärken sowie neuartige zu schaffen (EIF 35).

Darin untergräbt modernes Marketing die im Gedanken der Konsumentensouveränität anklingende Autonomie zweifach; einmal und ganz offensichtlich auf der Ebene der einzelnen Kaufentscheidung: Wenn etwa ein einkommensschwacher Bürger zu der Überzeugung gelangt, seine begrenzten finanziellen Mittel statt auf gesunde Nahrung lieber in eine Designersonnenbrille $\mathrm{zu}$ investieren, dann befriedigt er damit auf Kosten vitaler Bedürfnisse ein künstlich geschaffenes Verlangen. Die neoklassische Ökonomik findet das unproblematisch, denn aufgrund ihrer Abstraktion von allen qualitativen Wertfragen stellt sie künstlich erzeugte Bedürfnisse allen anderen gleich (Stigler/Becker 1977: 76-90; dagegen: Boulding 1987: 10-19). Doch selbst wenn deskriptiv der Konsum von Luxusgütern verglichen mit Gütern des Grundbedarfs subjektiv gleiche Befriedigungskurven zeitigte (was allerdings nur vereinzelt in massiv von Werbung überzogenen Gesellschaften der Fall ist), macht dies die Frage keineswegs obsolet, ob die Gesellschaft die eine Befriedigung der anderen normativ 
gleichstellen solle (Boulding 1989: 3-20, bes. 18); besonders dann nicht, wenn diese Art der Luxusgüterbefriedigung auf Kosten öffentlicher Güter geht bzw. hohe Opportunitätskosten mit sich bringt (AS 128). ${ }^{12}$ Die neoklassische Argumentation übersieht nämlich nonchalant den steil abfallenden Grenznutzen der nur durch Werbung absetzbaren Produkte, denn ,,since the demand [...] would not exist, were it not contrived, its utility or urgency, ex contrivance, is zero.“ (AS 131) Die Produktion an sich „unnützer“" Güter steht indes im Widerspruch zum neoklassischen Postulat, dass eine ungehemmte Wirtschaftstätigkeit ganz automatisch zur maximalen Effizienz der gesellschaftlichen Ressourcennutzung führt (AS 129).

Aber noch auf einer anderen Ebene und weit subtiler beeinträchtigt das ungehemmte Einwirken von Suggestivwerbung die individuelle wie gesellschaftliche Autonomie; insofern nämlich, als es die gesellschaftlichen Voraussetzungen vernünftiger Selbstorientierung insgesamt angreift. Die eigenen Präferenzen kritisch zu evaluieren, ist schließlich kein angeborenes, sondern ein kontingentes Vermögen des mündigen Subjekts, das durch kulturelle Formen begünstigt oder beeinträchtigt werden kann. Nun lässt sich aber über den Zuwachs an Lebensqualität durch eine immer weiter ausdifferenzierte Produktquantität wenig Wahres sagen, also hat die zum Kaufen verführen müssende Werbung eben viel zu lügen. „Social distinction must be associated with [...] a swimming pool, sexual fulfilment with a particular shape of automobile, social acceptance with a hair oil or mouthwash, improved health with a hand lotion [...]." (EPL 104)

Dergleichen „Wahrheiten“ sind nicht von selbst eingängig, darum müssen sie mit allen Mitteln der Suggestion eingeflößt werden. Eine Gesellschaft jedoch, in der ein an Wahrhaftigkeit ausgerichteter Diskurs um das gute Leben an den Rand gedrängt wird, weil alle öffentlichen Räume von Botschaften überflutet werden, die im Konsumieren die Antwort auf alle Lebensfragen versprechen, untergräbt die kulturellen Voraussetzungen moralischer und politischer Autonomie. ${ }^{13}$ Der Kapitalismus transformiert - und nicht selten pervertiert - die individuelle Freiheit, der er huldigt. Zudem ruiniert die Verstellung aller natürlichen wie künstlichen Räume mit Konsumappellen die Chancen einer in humaner Ästhetik gestalteten Lebensumwelt. Nicht zufällig, meint Galbraith, sind amerikanische Städte so hässlich; denn sie unterwerfen sich rückhaltlos dem Diktat kommerzieller Bedürfnisse, bequemen Konsums und effektiver Produktbewerbung (EPL 104).

12 Aus diesem Grunde halte ich die Galbraith von Jean Baudrillard entgegengebrachte Kritik, dieser folge einem naiv-naturalistischen oder/und einem gleichermaßen naiv-idealistischen Wertbegriff, für verfehlt (Baudrillard 1970: 17-26). Galbraith argumentiert als Beschreiber ökonomischer oder politischer Realitäten nirgends wertnaturalistisch. Davon zu unterscheiden sind seine in normativ-ethischer Hinsicht angestellten und in der Tat ,idealistischen“ Überlegungen zur Wirtschaftspolitik. In seiner Rolle als Ratgeber einer sich diskursiv konstruierenden politischen Öffentlichkeit, empfiehlt Galbraith selbstverständlich, was er für gut und richtig erachtet - in diesem Fall eine Anlehnung an biologisch gegebene Bedürfnisse. Konkret: Er tritt dafür ein, in der gesellschaftlich zu erarbeitenden Prioritätsordnung für die Wirtschaft das Beheben von Hunger und Not als ein gegenüber dem Verlangen nach Luxusgütern vorrangig zu befriedigendes Bedürfnis anzuerkennen. Einen ähnlichen Begriff humaner Grundbedürfnisse verwendet bereits Keynes 1931: 358-373.

13 Zustimmend und das Galbraith'sche Argument ausweitend Lippke 1989: 35-58; ähnlich Crisp 1987: 413-418. 


\section{Claus Dierksmeier}

Im Ergebnis heißt das, aufgrund der Strukturnotwendigkeiten moderner Massenproduktion versuchen die Unternehmen, den Konsumenten und (wie der nächste Abschnitt zeigen wird) auch den Staat zur Befriedigung von Bedürfnissen zu veranlassen, die ohne Not geschaffen wurden - eine bedenkliche Verschwendung von Ressourcen, ein hässlicher Troll im Gewande wirtschaftlicher Freiheit. Zwar begehrt der Bürger immer lauter, vor ungewollter Beeinflussung, gefährlichen Produkten, vermeidbarer Umweltbelastung und der Einspannung in unnötige Produktionsprozesse geschützt zu werden. Doch ironischerweise wird das gerade von einer Ökonomietheorie ignoriert, die vorgeblich ganz und gar im Interesse des Individuums steht. Die Neoklassik deutet das explizite Schutzverlangen des Bürgers kurzerhand als Wunsch nach Paternalismus um, dem nachzugeben - so Mises, Hayek, Friedman u. a. - eine unzulässige Abkehr vom Prinzip der Freiheit bedeute. Der bewusste Gebrauch, den die Bürger von ihrer Meinungsäußerungsfreiheit machen, wird also abgewertet im Namen eines ihnen bloß unterstellten (und von vielen sehr bewusst bestrittenen) unbewussten Verlangens nach grenzenlosem Konsum.

Mit diesem Schildbürgerstreich entzieht sich die neoklassische Theorie indes ihre eigene Legitimationsgrundlage. Zu sagen, der Bürger segne den ihm aufgedrängten Konsum mit seinen Kaufentscheidungen ab, da er mit diesen ja affirmativ auf die Reize der Werbung reagiere, heißt, spottet Galbraith, eben soviel, wie einen Mann mit einer Axt zu Boden zu strecken und dann zu behaupten, dies sei offensichtlich, wonach er sich gesehnt hätte, schließlich habe er ja deutlich auf den ihm verabreichten Impuls reagiert (NIS 349). Um tatsächliche Souveränität von Konsument und Bürger zu erwirken, ist es stattdessen wichtig, zuallererst den Mythos aufzuheben, dass das bestehende Wirtschaftssystem auf echter Freiheit und Konsumentensouveränität gründet (NIS 218). Galbraiths Kritik der neoklassischen Mikroökonomik kulminiert also in der Forderung nach einer wirtschaftsphilosophischen Selbstaufklärung der Gesellschaft.

Solange die Illusion besteht, dass das Wirtschaftsystem in sich selbst neutral ist und in seiner konkreten Gestalt lediglich die aggregierten Freiheitsentscheidungen aller beteiligten Individuen ausdrückt, kommt den bestehenden Verhältnissen eine pseudoliberale Scheinlegitimität zu. Und diese steht politischen Reformen im Weg, zum Nachteil des Gemeinwohls - sowie zum Vorteil einiger weniger Privilegierter. Anstatt indes Freiheit rein quantitativ als „maximum range of choice“ zu verstehen (NIS 217), verfolgt Galbraith einen qualitativen Freiheitsbegriff, welcher der Diktatur des Kommerz entgegentritt, wie ein näherer Blick auf die Wirtschaftspolitik der Gegenwart schnell verdeutlicht. ${ }^{14}$

\section{Galbraiths Kritik der neoliberalen Wirtschaftspolitik}

Weil das moderne Unternehmen systembedingte Eigeninteressen verfolgt, die nicht automatisch mit dem Gemeinwohl konvergieren, bedarf es einer wirtschaftswissenschaftlichen Theorie, welche die real existierende unternehmerische Freiheit begreift. Entgegen der Neoklassik sind Unternehmen keine Maschinen, deren Algorithmen außerhalb ihrer selbst zu analysieren und zu optimieren wären. Unternehmen sind vielmehr selbstbezügliche Ent-

14 Sen 1999: 139-146, bes.141; Heilbronner 1999: 100-103; Marglin 1999: 130. Zur terminologischen Unterscheidung zwischen quantitativer und qualitativer Freiheit Dierksmeier 2007: 107-119. 
scheidungssysteme, die ideengeleitet - und mithin wirtschaftsethisch bewertbar - handeln. Das lässt sich gut illustrieren am Bestreben des Managements, Planungsunsicherheiten durch Nachfragemanipulation zu minimieren.

Marketing zielt oftmals nicht nur in Richtung auf den individuellen Kunden als Abnahmegaranten, sondern auch auf den Staat und die Gesellschaft insgesamt. Gefährlicher noch als ein Schwanken der Kaufneigungen vereinzelter Marktteilnehmer ist ein Wegbrechen der gesamtgesellschaftlichen Nachfrage aufgrund von Rezession, Inflation etc. Also liegt es im unternehmerischen Interesse, die gesamtgesellschaftliche Kaufkraft und zu diesem Zweck letztendlich die staatliche Wirtschaftspolitik insgesamt zu beeinflussen. Insbesondere hochtechnisierte industrielle Großunternehmen suchen die Nähe zum Staat: Sind Kapitalinvestitionen hoch und ist der Produktionserfolg unsicher - wie etwa bei neuen Rüstungstechnologien - mag sich der Hersteller nur ungern auf das Risiko einer ausbleibenden Abnahme einlassen. Wer ein komplexes Raketenabfangsystem produziert, muss bei Strafe des Bankrotts wissen, dass er dafür einen sicheren Abnehmer hat. Man muss daher von Unternehmerseite mit allen erlaubten (und gegebenenfalls unerlaubten) Mitteln sicherstellen, dass der Staat langfristig eine vorhersehbare und möglichst steigende Gütermenge abnimmt (CC 136). Intime personelle Verflechtungen von Schwer- und Rüstungsindustrie mit der Politik bis hin zur Mitgestaltung der Außenpolitik seitens der industriellen Entscheider sind naheliegende Konsequenzen (EIF 54).

Ganz ähnliche Verflechtungen von Privatwirtschaft und Staat sind auch in anderen technologie- und kapitalintensiven Sektoren zu beobachten (Fahrzeugindustrie, Bausektor). Anders als die Neoklassik, welche Staat und Privatwirtschaft kategorial trennt und deshalb vor ihrer faktischen Annäherung gerne die Augen verschließt, sieht Galbraith die Überlappungen des öffentlichen und des privaten Sektors als die Regel, nicht die Ausnahme moderner Wirtschaftspraxis an und will die Privatwirtschaft deshalb nicht in den politikfreien Raum entlassen (CC 75). Betrachten wir den volkswirtschaftlichen Hintergrund dieses Arguments etwas näher:

Konjunkturelle Krisen zeichnen die transformierten Strukturbedingungen der modernen Wirtschaft im Negativ nach. Einerseits ist es für jede technisch aufwendige Produktion vonnöten, dass sowohl seitens der Unternehmen wie seitens der Privathaushalte viel gespart wird, damit die zurückgelegten Summen durch den Kreditmarkt an die Unternehmen übergeben und kostenintensive Investitionen getätigt werden können. Aber je mehr gespart wird, umso größer ist auch die Hebelwirkung auf das Wirtschaftsgeschehen, wenn jene Gelder einmal nicht in den Wirtschaftszyklus zurückgespeist werden (NIS 222). Ein Großunternehmen kann auf reduzierte Nachfrage nur eingeschränkt preiselastisch reagieren; neben den beschriebenen produktionstechnischen Gründen liegt dies daran, dass der Aktienmarkt ein Absinken der Profitratio in aller Regel abstraft und dass so das Unternehmen proportional zu den Anteilen, die es an sich selbst hält, an Investiv- bzw. Deckungskapital verliert. Um Eigenkapital zu erhöhen, legt das Eigeninteresse des Managements es nahe, die Betriebskosten zu senken. Und das kann durch Produktionsverknappung sowie die Entlassung von Arbeitskräften erreicht werden. Betriebswirtschaftlich rationales Handeln führt also hier durchaus nicht zur optimalen Allokation aller Ressourcen, sondern zu volkswirtschaftlich manifest nachteiligem Verhalten: Durch Arbeitslosigkeit und ausbleibende Lohnzahlungen sinkt die gesell- 


\section{Claus Dierksmeier}

schaftliche Kaufkraft weiter ab, was den Trend, aus dem die ursprünglichen Absatzprobleme resultierten, noch verstärkt. Es kommt zu Unterbeschäftigungsgleichgewichten (Mankiw/ Romer 1991: 412; Iwand/Thomassen 1979: 331-351, bes. 339).

Galbraith verficht darum die Keynesianische Theorie einer antizyklischen Wirtschaftspolitik; er glaubt aber nicht, dass ad hoc konzipierte Arbeitsbeschaffungsmaßnahmen in solchen Fällen Abhilfe schaffen. Sie greifen zu langsam und, einmal ins Leben gerufen, lassen sich öffentliche Dienstleistungen nicht mehr so leicht aus der Welt schaffen. Genau das setzt Keynes aber voraus: dass nämlich im Falle inflationärer Überhitzung der Staat die Konjunktur auch künstlich bremsen kann. Geschieht dies nicht, kommt es langfristig zur Stagflation (Sachs/Michael 1985). Der Staat fährt darum besser, weil flexibler, meint Galbraith, wenn er mit dem leichter zu ändernden Steuersystem operiert. Dieses vermag - sofern progressiv aufgebaut - als automatisches Konjunkturkorrektiv zu wirken. Bei progressiven Steuern kommt es, wann immer besonders hohe Gewinne auftreten, zu einer übermäßigen Abschöpfung seitens des Staates; eine konjunkturelle Überhitzung durch fortlaufende Reinvestitionen wird somit erschwert. Bleiben die Gewinne hingegen aus, sinken umgekehrt die steuerlichen Belastungen der Unternehmer überproportional und gleichen deren Verlust an Investivkraft partiell wieder aus (CCC 182).

Um nun aber Steuereinnahmen vorhersagbar stetig wieder in den Wirtschaftszyklus zurückfließen zu lassen, braucht es einen üppig ausgestatteten öffentlichen Sektor. Die Geschichte gibt Galbraith mit dieser gegen alle neoklassische Lehre streitenden Annahme Recht: Die Zeiten im 20. Jahrhundert, in denen die öffentliche Hand intensiv im amerikanischen Wirtschaftsleben mitmischte (während des zweiten Weltkrieges und beim Wettrennen zum Mond), waren zugleich die Epochen, in denen die amerikanische Wirtschaft am stärksten prosperierte. Trotz starker Bedenken gegenüber der Gestaltung der Außenpolitik vonseiten der Rüstungsindustrie, lobt Galbraith jene konjunkturstabilisierende Wirkung der amerikanischen Rüstungsproduktion. Auch hebt er die im Zuge derselben ermöglichte technologische Forschung heraus, die aufgrund ihres enormen Kostenaufwandes in dieser Form von privater Seite nicht durchgeführt werden könnte, in ihren Resultaten aber auch der zivilen Wirtschaft zugutekommt.

Aus dem Erfolg jener öffentlich-privaten Gemeinwirtschaft im Rüstungssektor leitet Galbraith deshalb so ironisch wie konsequent ab, dieser Industriekomplex sei nunmehr zu verstaatlichen (EPP 284 f.). So ließe sich das Militärbudget der USA schrittweise reduzieren und in Investitionen für zivile Forschung umlenken, die zur Stabilisierung der Konjunktur ebenso taugt, außenpolitisch aber weniger bedenkliche Resultate zeitigt (NIS 229). Gibt man nämlich die neoklassische Illusion auf, es handele sich bei der mit dem Regierungshandeln intim verflochtenen Militärwirtschaft und Schwerindustrie um nichts weiter als etwas groß geratene Privatunternehmungen mit zufälligerweise stets gefüllten Auftragsbüchern (AP 142), so fällt eben auch die Konklusion dahin, der Staat dürfe sich keinesfalls einmischen (EIF 54 ff.). Was alle angeht, muss auch von allen angegangen werden. Galbraiths makroökonomische Aufklärung und ihre wirtschaftsphilosophische Auslegung dienen also dem Zweck, das Wirtschaftsleben demokratischer Kontrolle zu unterstellen.

Mit gleicher Zielsetzung hat Galbraith noch kurz vor seinem Ableben die großen Wirtschaftsskandale der letzten Jahre analysiert. Die in jenen Skandalen zum Vorschein gekom- 
mene unheilige Allianz von kreativen Buchhaltungskünstlern, achtlosen privaten Unternehmensprüfern und nachsichtigen öffentlichen Aufsichtsgremien konnte unbehelligt im Schlagschatten einer neoliberalen Politik operieren, die ihrerseits von der neoklassischen Wirtschaftstheorie inspiriert war (EIF 50). Denn Letztere lehnt kontrollierende Eingriffe ins Wirtschaftsgebaren per definitionem als freiheitswidrig und effizienzmindernd ab (EIF 8). Die augenfällige Koinzidenz zwischen der Deregulierung des amerikanischen SecuritySektors und dem darauf folgenden Savings-and-Loans-Debakel, zwischen der Deregulierung des Investment-Bankings und der sich anschließenden WorldCom-Pleite sowie zwischen der Deregulierung des Buchhaltungsrechts und des Enron/Arthur-Andersen-Skandals legt jedoch das gerade Gegenteil nahe. ${ }^{15}$ Regierung und Verwaltung verfahren keineswegs per se ineffizient, und umgekehrt stellen Unternehmen nicht per se produktive Gebilde dar, die man deshalb von jeder gesellschaftlichen Aufsicht entlasten muss. Weil aber den Vertretern der Neoklassik der freie Markt ,a semireligious totem“ ist (CC 135), verschließen sie die Augen davor, dass Unternehmen nur unter bestimmten und keineswegs immer gegebenen Voraussetzungen produktiv und gemeinwohlförderlich operieren. Dem Ansehen des Kapitalismus schadet daher nichts so sehr wie die Regentschaft seiner Apologeten, resümiert Galbraith (M 312).

Ein weiteres Beispiel für wirtschaftspolitische Fehlgriffe basierend auf der neoklassischneoliberalen Doktrin ist der Versuch, Unternehmenssteuern so weit wie möglich zu senken, damit die marginalen Kosten der Produktion fallen - und mehr Güter hergestellt werden können. Das Aufkommen an Unternehmenssteuern in den USA befindet sich mittlerweile auf dem niedrigsten Stand seit über fünfzig Jahren. Entsprechend fehlt Geld für öffentliche Aufgaben und den Abbau von Armut. Dennoch verteilten die jüngsten Steuerreduktionen in den USA einmal mehr Geld an ,those who would not spend“, und hielten nichts als ,privation“ bereit „for those who would“ (EIF 59); sie waren mithin nicht nur sozial unausgewogen, sondern vor allem auch volkswirtschaftlich „manifestly ineffective“ (EIF 44; dazu auch Cornia 2004: 3-26). Rezessionsrisiken werden dabei wissentlich in Kauf genommen. Den finanzschweren Institutionen, an deren Bilanzwerten sich diese Politik ausrichtet, können solche Krisen wenig anhaben. Hingegen reduzieren die zu Rezessionszeiten verabreichten Kuren - „vigorous downsizing“ durch „layoffs of those least responsible“ (EIF 42) - das Vermögen der arbeitenden Bevölkerung.

Anstelle einer antizyklischen Fiskalpolitik, die direkt den Bürgern nützt und nur indirekt (über eine steigende Gesamtnachfrage) den Unternehmen, präferiert die neoklassisch-neoliberale Agenda eine monetaristische Wirtschaftspolitik, welche direkt allein den Unternehmen dient (Planungssicherheit) und verspricht, dass deren gesteigerter Wohlstand indirekt zur Bevölkerung herabsickert (trickle down-economics) (DeLong 2000: 83-94). Im Streit über Fiskalismus versus Monetarismus in der Wirtschaftspolitik kommt es erneut zum Konflikt zwischen dem von Galbraith vertretenen Freiheitsbegriff, der nach der Wertigkeit der gesellschaftlich erzeugten Freiheit fragt, und dem bloß quantitativen Freiheitsbegriff der Neoklassik. In monetaristisch geführten Ökonomien verlieren die ärmeren Schichten nicht nur kontinuierlich an relativem Einkommen, sondern damit Zug um Zug auch an gesellschaftspolitischem Gewicht im Wettstreit um die öffentliche Meinung gegenüber der

15 Bailey/Harte/Sugden 2000: 197-218; Vinten 2002: 4-9; Baker 2003: 446-466. 


\section{Claus Dierksmeier}

aktiv Politikfinanzierung und Meinungsmache betreibenden Firmenwelt (EPP 255). Galbraith findet es darum zuhöchst zynisch, wenn monetaristische Politik unter der Fahne wirtschaftlicher Freiheit gegen eine fiskalistische Sozialpolitik antritt. „Income rising above the level of mere subsistence is [...] a liberating force. [...] It is one of the curiosities of much social comment that such welfare measures are regularly seen as limitations on freedom - the freedom presumptively inherent in the free enterprise system." (AP 51)

Ein ernsthafter Liberalismus muss zuerst der Freiheit der ökonomisch Schwachen verpflichtet sein (CCC 151); die Wohlhabenden können sich schon selbst Gehör und Einfluss verschaffen. Eine der Hauptaufgaben des Staates ist es darum, allen „countervailing powers“ aufzuhelfen (CCC 128), die gegen Machtasymmetrien am Markt ankämpfen. Dies sind Gewerkschaften, Genossenschaften, kooperative Unternehmungen, wohltätige Vereine, etc.; aber eben auch rechtliche Institutionen, wie das Recht auf ein Mindesteinkommen (EPP 262), welche den am Markt unterrepräsentierten Kräften aufhelfen. Doch, so notiert Galbraith frustriert, eben weil „,social security“ den Armen Sicherheit gibt und ihnen damit politisch den Rücken stärkt (AP 187), wird sie von einer Mehrheit der „contented and [...] comfortable“ (CC 144) bekämpft. Mit Segnung der Mehrheitsdemokratie wird in den USA gesellschaftliche Armut verstetigt und die „,social balance“ verspielt ${ }^{16}$; „,private opulence“ und „public squalor" bzw. ,public penury and private affluence“ kontrastieren immer krasser (EPL 93; vgl. auch Karier 1993: 23-28). Der Grund dafür liegt letztlich in wirtschaftsphilosophischen Meinungsverschiedenheiten: „The difference between Americans and Europeans is not that Americans have a peculiar ineptitude for operating public enterprises. The difference is that Americans have been guided by a doctrine that accords a second-rate and apologetic status to such effort." (EPP 281)

Noch einmal also: Wenn ihnen die Wettbewerbshüter nicht auf die Finger schauen, neigen Unternehmer (wie bereits Adam Smith beklagte) dazu, durch verdeckte Preisabsprachen und - sofern sie die Politik auf ihre Seite bringen können - durch Verzerrungen der internationalen Preisgefüge (Subventionen, Einfuhrbeschränkungen) ungeliebten Konkurrenzdruck zu vermindern. Unternehmen haben also, solange man ihr betriebswirtschaftliches Kalkül nicht gezielt von außen beeinflusst, die unschöne Tendenz volkswirtschaftlich ineffizient zu operieren - und überdies die persönliche wie politische Freiheit der Bürger zu unterminieren. Angesichts allenthalben oligopolisch und nicht streng-kompetitiv geprägter Märkte verkehrt sich der neoklassisch-neoliberale Einwand gegen demokratisch-partizipative Wirtschaftsgestaltung von daher in sein Gegenteil: Nur im Rahmen einer kritischen Wirtschaftsaufsicht führt privates Erwerbsstreben zu gesellschaftlichen Wohlfahrtsgewinnen. Die - auch ökonomische - Freiheit der Bürger wird durch wirtschaftspolitische Regulierungen geschützt, nicht verkürzt.

Politik sollte dafür sorgen, dass Unternehmen die von ihnen produzierten negativen externen Effekte internalisieren. In der Tat kann Ordnungspolitik - vermittels Verbrauchssteuern und Emissionsabgaben - dazu beitragen, dass Unternehmen die öffentlichen Kosten ihres Produktionsprozesses in ihre Bilanz aufnehmen und so zu einem sorgsameren Umgang mit

16 ,The present age of contentment will come to an end only when and if the adverse developments that it fosters challenge the sense of comfortable well-being [...] widespread economic disaster, adverse military action that is associated with international misadventure, and eruption of an angry underclass." (CC $156 \mathrm{f}$.) 
der Umwelt angetrieben werden. Dabei muss sich eine Gesellschaft allerdings vor Augen führen, dass das Sprachspiel der Internalisierung von Externalitäten eines nicht zum Ausdruck bringt: Wenn lediglich der Markt als Allokationsmechanismus thematisiert wird, dann impliziert das allein schon einen Verzicht auf all jene Güter, die am Markt selbst unter optimalen Rahmenvorgaben nicht gehandelt werden können. Lokale Umweltschäden lassen sich (mit hohem taxometrischen Aufwand) noch einigermaßen quantifizieren und somit durch staatlichen Druck in unternehmerische Kostenkalküle implementieren; aber wie steht es um Güter, deren Wesensart eine solche Verrechnung nicht zulässt?

Die Antwort, das derartige Güter dann eben von staatlicher Seite aus geschaffen werden müssen, ist so richtig wie ungenügend, sofern die Möglichkeiten des (nationalstaatlich regulierbaren) Rechts hinter den Wirklichkeiten der (global operierenden) Wirtschaft zurückbleiben. Eine globalisierte Wirtschaftspraxis überstrapaziert die Lösungskompetenz nationaler Politik nicht zufällig, sondern strukturell. Und genau an dieser theoretischen Sollbruchstelle - wo anderen Theorien nur die angesichts der derzeitigen weltpolitischen Lage unglücklicherweise utopische Forderung nach einem sanktionsbewehrten Welt(wirtschafts)recht bleibt - offeriert Galbraiths Theorie überraschende und bisher noch ungenutzte Potenziale (Dunn 2005: 90-112; Adams 1984: 91-102).

Galbraiths mikroökonomische Studien haben gezeigt, dass managementgeleitete Unternehmen sich durchaus nicht notwendig in Opposition zu Arbeitnehmerschaft und Öffentlichkeit befinden müssen. Da die Nachhaltigkeit der eigenen Tätigkeit im aufgeklärten Selbstinteresse des Unternehmens liegt, hat das Management vielmehr handfeste Anreize, die Zielvorgaben seiner wirtschaftsethisch informierten Kunden und Stakeholder zu berücksichtigen. Derlei vermeidet schließlich Prozesskosten, verhindert Reputationsschäden und erhöht den Kooperationsnutzen eines Unternehmens, und all das wirkt sich letztendlich auch auf die Bilanz aus. Zudem verschafft die nötige soziale Anerkennung einen höheren Kapitalzufluss, denn der Erfolg des modernen Managements wird auch und gerade am Kapitalmarkt zusehends danach evaluiert, inwieweit sich die Unternehmenspraxis mit denn allgemeinen gesellschaftlichen Wertvorstellungen in Einklang befindet. Galbraith liefert also mit seiner systemisch ausgerichteten Theorie des modernen Großunternehmens nicht nur en passant wichtige theoretische Grundlagen für die Nachhaltigkeitsdebatte. Seine Theorie erklärt auch, warum Firmen vom Grundsatz kurzfristiger Profitmaximierung zugunsten einer langfristig ausgerichteten triple bottom line (sozial, ökologisch und ökonomisch) abrücken können (Schäfer 2001).

Der komparative Vorteil dieser Position lässt sich gut an einem Paradebeispiel der Unternehmensethik, dem Isolationsparadox, verdeutlichen: Es ist eine Binsenwahrheit herkömmlicher business ethics, dass Firmen ethischen Impulsen operational genau dann nicht nachgehen, wenn isoliert-moralisches Handeln ihre Ausmerzung durch Konkurrenz zur Folge hätte. Statt nun einfach ihre Ohnmacht zu deklarieren und die Hände in den Schoß zu legen, suchen zusehends mehr multinationale Unternehmen aktiv nach Auswegen in solchen Situationen - mit dem erklärten Ziel, strukturelle Anreize zur Verletzung ethischer Unternehmerpflichten auszumerzen. So verabreden einige Großunternehmen mit der Konkurrenz freiwillige Selbstkontrollen entlang globaler Standards, deren Einhaltung dann seitens des NGO-Sektors und durch die Organisation öffentlicher Aufmerksamkeit abgestützt wird. 


\section{Claus Dierksmeier}

Andere Unternehmen betreiben Lobbyismus, um internationale Regelwerke herbeizuführen, die sicherstellen, dass moralisches Unternehmerhandeln am Markt bestehen kann. Wieder andere verbünden sich mit der Öffentlichkeit und nutzen den Umweg über die ethische und/oder ökologische Zertifizierung ihrer Produkte, um trotz nachhaltiger Produktionsmethoden und somit erhöhter Preise am Markt bestehen zu können. ${ }^{17}$

So wurden im Rahmen proaktiver Corporate Social Responsibility in den vergangenen Jahren ungeheure Fortschritte gemacht, die bislang von der akademischen Wirtschaftsethik noch nicht zureichend reflektiert und systematisiert wurden. Die neoklassisch-neoliberale Doktrin - auf den Punkt gebracht in Milton Friedmanns Satz, die wirtschaftsethische Pflicht des Unternehmens sei einzig und allein, Profit zu machen ${ }^{18}$ - kann eine Realisierung wirtschaftsethischer Ideale ja nur insoweit erklären, als deren Wirkungen sich direkt positiv im Budget niederschlagen. Das wird zwar häufig langfristig, aber nicht immer und nur selten kurzfristig der Fall sein (Webley/More 2003 [m. w. N.]). Jede nicht buchhalterisch zu verrechnende unternehmerische Anstrengung für eine bessere Welt muss (neoliberal) als den rechtlich gesicherten Interessen der Anteilseigner zuwider und (neoklassisch) als ökonomisch unvernünftig abgemahnt werden. Dennoch geschieht es: Einem weiten Feld von unternehmerischen Bestrebungen zum Corporate Citizenship ist es zu danken, dass es im Verbund mit der UNO, der ILO und der WTO mitunter gelang, einige der besonders eklatanten Regelungslücken im internationalen Wirtschaftsrecht zu schließen.

Die Initiativen der Zivilgesellschaft und der Wirtschaft zur Linderung des Armutselends können staatliches Handeln natürlich nicht vollauf ersetzen. Die ungeheuren Ungleichheiten in der globalen Vermögensverteilung verlangen vielmehr dringend nach einer koordinierten Initiative der wohlhabenden Nationalstaaten. Die Allianz aus öffentlicher Meinung und aufgeklärtem unternehmerischen Selbstinteresse kann staatliche Weltwirtschaftspolitik schon deshalb nicht überflüssig machen, weil Rechtszwang die ultima ratio aller freiheitlichen Ordnungen darstellt. Wo Politik die Bedingungen für Armut geschaffen hat, indem sie vorhandene Governance-Strukturen zerstörte, muss sie auch die Verantwortung für die Wiederherstellung des Rechts akzeptieren. Das gilt für weite Teile der Dritten Welt, wo eine Diktatur der Großkonzerne durch voraufgehendes Kolonialunrecht und die damit einhergehende imperialistische Zerstörung autochthoner politischer Gefüge ermöglicht wurde. Den so von den Industrienationen selbst herbeigeführten regellosen Zuständen unter Verweis auf die Grenzen nationaler Politik tatenlos zuzusehen, ist in Galbraiths Augen nur eine hohle ,formula for selfishness“"19.

Um den benachteiligten Nationen der Erde ökonomisch aufzuhelfen (GS 135), müssen sich die wohlhabenden Nationen letztendlich zum Aufbau einer sozial wie ökologisch ausgewogenen Weltwirtschaftsordnung bereitfinden. Indes liegt die Meinungsführerschaft zugunsten einer solchen Weltinnenpolitik heute weniger bei der Staatenwelt als vielmehr bei einer in alle wirtschaftlichen und politischen Nischen schauenden engagierten Öffentlichkeit

17 Siehe die Norms on the Responsibilities of Transnational Corporations and Other Business Enterprises with Regard to Human Rights, U.N. Doc. E/CN.4/Sub.2/2003/12/Rev.2 (2003); zur Global Compact-Initiative der UN http://www.un.org/Depts/ptd/global.htm.

18 Friedman, Milton, in: The New York Times Magazine, 13.09.70.

19 GS 131; siehe auch Reuss 1989: 151-160; Galbraith 1989: 231-253. 
im Verbund mit Unternehmen, die in augenscheinlichem Ernst und mit augenfälligem Erfolg mehr und mehr, aller neoklassischen Ökonomik zuwider, ihrer Verantwortung als Korporationen im öffentlichen Leben gerecht werden und dafür am Markt von mündigen Konsumenten präferiert werden. Die Stärke von Galbraiths Position liegt darin, dass er ein entsprechendes Zusammenwirken von Wirtschaft und Gesellschaft nicht nur abstrakt eingefordert, sondern konkret vorgezeichnet hat. Mit Galbraith lässt sich zeigen, dass Unternehmer, Konsumenten und staatliche wie zivile Akteure über hinreichende wirtschaftliche Entscheidungs- und Handlungsspielräume verfügen, um der Pflichtseite des Begriffs wirtschaftlicher Freiheit gerecht zu werden. Seine Wirtschaftsphilosophie ermöglicht es, die notwendige Verantwortung für unsere ökologische und soziale Lebenswelt mit den Prinzipien der freiheitlichen Markt- und Verkehrswirtschaft zusammenzudenken.

\section{Zusammenfassung}

Ausgehend von seiner Kritik des physikalistischen Selbstverständnisses der Wirtschaftswissenschaften plädiert Galbraith dafür, Ökonomik als selbstkritische Sozialwissenschaft zu betreiben. Als ein Theoriegefüge, von dem Handlungsempfehlungen abgeleitet werden, trägt die Ökonomik zur Realisierung ihres eigenen Wirklichkeitsbildes bei und muss sich daher dem gesellschaftlichen Wertediskurs öffnen. Solange die Wirtschaftswissenschaft ihren wirklichkeitsgestaltenden Einfluss nicht reflektiert, wird sie bestenfalls - sofern an gegenwärtigen Phänomenen orientiert - konservativ, schlimmstenfalls aber - sofern an überkommenen Prämissen ausgerichtet - reaktionär wirken. Soll die sozioökonomische Wirklichkeit indes korrekt begriffen und angemessen auf sie reagiert werden, ist ein Wechsel des vorherrschenden materialistischen Paradigmas in der Ökonomik vonnöten.

Die Chance für einen solchen Neuansatz erblickt Galbraith in den durch die multinationalen Großunternehmen veränderten ökonomischen Handlungs- und Entscheidungsstrukturen. Angesichts der organisierten Macht der Konzerne zur Marktverzerrung und zur Beeinflussung öffentlicher Entscheidungen - und der entsprechend geschwächten Position des Individuums und des Staats - lassen sich etliche der Gleichgewichtspostulate der neoklassischen Ökonomik nur noch sehr eingeschränkt aufrechterhalten. Eine diesem Strukturwandel gerecht werdende Wirtschaftswissenschaft kann nicht länger nur dem Markt als Allheilmittel vertrauen. Es gilt vielmehr durch Wirtschaftspolitik und Corporate Social Responsibility, jene Verhältnisse abzubauen, in denen vorgeblich im Namen der Freiheit den Menschen - gegen deren ausdrücklichen Willen - die Grundlagen eines lebenswerten Lebens Schritt für Schritt entzogen werden. Die Relevanz des Lebenswerks von John Kenneth Galbraith besteht darin, eine solche neue Ökonomik vorangetrieben, sie in verständlichen Kategorien dargestellt und so die Möglichkeitsbedingungen einer sowohl freien wie gerechten und ökologisch nachhaltigen Weltwirtschaft skizziert zu haben. 


\section{Claus Dierksmeier}

Verzeichnis der verwendeten Siglen von John Kenneth Galbraiths Werk

$\begin{array}{ll}\text { AAL } & \text { Annals of an Abiding Liberal, New York } 1979 . \\ \text { AC } & \text { American Capitalism: The Concept of Countervailing Power, New York } 1993 \\ & \text { (Original 1952). } \\ \text { AP } & \text { The Anatomy of Power, New York 1985. } \\ \text { AS } & \text { The Affluent Society, New York 1958. } \\ \text { AU } & \text { The Age of Uncertainty: Points of Departure, New York 1978. } \\ \text { CC } & \text { The Culture of Contentment, New York 1993. } \\ \text { CCC } & \text { Capitalism, Communism, Coexistence: From the Bitter Past to a Better Prospect, } \\ & \text { New York 1988. } \\ \text { ED } & \text { Economic Development, Cambridge 1964. } \\ \text { EIF } & \text { The Economics of Innocent Fraud: Truth for our Time, New York 2004. } \\ \text { EPL } & \text { A Contemporary Guide to Economics, Peace and Laughter, New York } 1971 . \\ \text { EPP } & \text { Economics and the Public Purpose, New York 1973. } \\ \text { GC } & \text { The Great Crash 1929, New York (Original 1954). } \\ \text { GS } & \text { The Good Society: The Humane Agenda, New York 1996. } \\ \text { LT } & \text { A Life in our Times: Memoirs, New York 1983. } \\ \text { M } & \text { Money, Whence it Came, Where it Went, New York 1975. } \\ \text { NIS } & \text { The New Industrial State, New York 1986 (Original 1971). } \\ \text { VS } & \text { A View from the Stands: Of People, Politics, Military Power, and the Arts, } \\ & \text { New York 1986. }\end{array}$

\section{Sekundärliteratur}

Adams, John, 1984: Galbraith on Economic Development, in: Journal of Post-Keynesian Economics 7 (1), 91-102.

Backhaus Jürgen/Hansen Reginald, 2000: Methodenstreit in der Nationalökonomie, in: Journal for General Philosophy of Science 31 (2), 307-336.

Bailey, David/Harte, George/Sugden, Roger, 2000: Corporate disclosure and the deregulation of international investment, in: Accounting, Auditing \& Accountability Journal 13 (2), 197-218.

Baker, Richard, 2003: Investigating Enron as a public private partnership, in: Accounting, Auditing \& Accountability Journal 16 (3), 446-466.

Baudrillard, Jean, 1970: La Société de consommation: ses mythes, ses structures, Paris.

Bergmann, Barbara R., 1989: Why do most economists know so little about the economy?, in: Bowles/Edwards/Shepherd 1989, 29-37.

Boulding, Kenneth E., 1969: Economics as a Moral Science, in: American Economic Review 59 (1), 1-12.

Boulding, Kenneth E., 1987: The Economics of Pride and Shame, in: Atlantic Economic Journal 15 (1), 10-19. 
Boulding, Kenneth E., 1989: The Pathologies of Persuasion, in: Bowles/Edwards/Shepherd 1989, 3-20.

Bowles, Samuel/Edwards, Richard C./Shepherd, William G. (Hrsg.), 1989: Unconventional Wildom: essays on economics in honor of John Kenneth Galbraith, Boston.

Caves, Richard, 1970: Uncertainty, Market Structure and Performance: Galbraith as Conventional Wisdom, in: Jesse W. Markham/Gustav F. Papenek (Hrsg.), Industrial Organization and Economic Development, New York, 283-302.

Chamberlain, Edward H., 1948: An experimental imperfect market, in: Journal of Political Economy 56, 95-108.

Colander, David/Klamer, Arjo, 1987: The Making of an Economist, in: Journal of Economic Perspectives 1 (2), 95-11.

Cornia, Giovanni Andrea, 2004: Inequality, Growth, and Poverty: An Overview of Changes over the Last Two Decades, in: ders. (Hrsg.), Inequality Growth and Poverty in an Era of Liberalization and Globalization, Oxford, 3-26.

Crisp, Roger, 1987: Persuasive Advertising, Autonomy, and the Creation of Desire, in: Journal of Business Ethics 6, 413-418.

Cyert, Richard M./March James G., 1992: A Behavioral Theory of the Firm, Englewood Cliffs (1. Aufl. 1963).

DeLong, John Bradford, 2000: The Triumph of Monetarism?, in: Journal of Economic Perspectives 14 (1), 83-94.

Demsetz, Harold, 1970: Economies in the Industrial State: Science and Sedative: Diskussion, in: American Economic Review 60 (2), 481-484.

Dierksmeier, Claus, 2007: Quantitative oder qualitative Freiheit?, in Paolo Becchi u. a. (Hrsg.), Nationen und Gerechtigkeit, Rechtsphilosophische Hefte XII, Frankfurt a. M., 107-119.

Dunn, Stephen, 2005: John Kenneth Galbraith and the Multinational Corporation, in: Challenge. Magazine of Economic Affairs 48 (2), 90-112.

Friedman, Milton, 1953: The Methodology of Positive Economics, in: ders., Essays in Positive Economics, Chicago.

Galbraith, James, 1984: Galbraith and the theory of the corporation, in: Journal of PostKeynsian Economics VII (1), 43-60.

Galbraith, James, 1989: Trade and the planning system, in: Bowles/Edwards/Shepherd 1989, 231-253.

Heilbronner, Robert, 1999: Ken Galbraith as worldly philosopher, in: Sasson 1999, 100-103.

Iwand, Thomas/Thomassen, Henry, 1979: Galbraith's Theory of the Mature Corporation, in:

Theory and Decision 10,331-351.

Karier, Thomas, 1993: The heresies of John Kenneth Galbraith, in: Challenge. Magazine of Economic Affairs 36 (4), 23-28.

Keynes, John Maynard, 1931: Economic Possibilities for Our Grandchildren, in: Essays in Persuasion, London, 358-373.

Laperce, Blandine/Uzunidis, Dimitri, 2006: John Kenneth Galbraith and the Future of Economics, New York.

Leibenstein, Simon, 1987: Inside the Firm: The Inefficiencies of Hierarchies, Cambridge/MA. Lippke, Richard L., 1989: Advertising and the Social Conditions of Autonomy, in: Business 


\section{Claus Dierksmeier}

\& Professional Ethics Journal 8 (4), 35-58.

Lowry, S. Todd, 1987: The archeology of economic ideas. The classical Greek tradition, Durham.

McCloskey, Donald, 1994: Knowledge and Persuasion in Economics, New York.

Mankiw, N. Gregory/Romer, David (Hrsg.), 1991: New Keynesian Economics, Vol. I, Cambridge/MA.

Marglin, Stephen, 1999: John Kenneth Galbraith and the myths of economics, in: Sasson 1999, 114-138.

Marris, Robin, 1964, The Economic Theory of Managerial Capitalism, London.

Marris, Robin, 1968: Galbraith, Solow and the Truth About Corporations, in: Public Interest 11, 37-45.

Marshall, Alfred, 1890: Principles of Economics, London.

Menninger, Karl, 1969: Number Words and Number Symbols. A Cultural History of Numbers, Cambridge.

Niehans, Jürg, 1990: A History of Economic Theory: Classic Contributions 1720-1980, Baltimore. Parker, Richard, 2005: John Kenneth Galbraith. His Life, His Politics, His Economics, New York.

Reich, Robert B., 1999, Galbraith in the New Gilden Age, in: Sasson 1999, 88-100.

Reuss, Henry S., 1989: Reuniting economics with politics, in: Bowles/Edwards/Shepherd 1989, 151-160.

Robbins, Lionel, 1932: An Essay on the Nature and Significance of Economic Science, New York.

Roll, Eric, 1989: What is economics? What is an economist? The Case of J. K. Galbraith, in: Bowles/Edwards/Shepherd 1989, 113-122.

Sachs, Jeffrey/Michael, Bruno, 1985: The economics of worldwide Stagflation, Cambridge 1985.

Sahlins, Marshall D., 1972: Stone Age Economics, New York.

Samuels, Warren J., 1984: Galbraith on economics as a system of professional belief, in: Journal of Post-Keynesian Economics VII (1), 67-76.

Sasson, Helen (Hrsg.), 1999: Between Friends. Perspectives on John Kenneth Galbraith, Boston/New York.

Say, Jean-Baptiste, 1803: Traité d'économie politique, ou simple exposition de la manière dont se forment, se distribuent, et se composent les richesses, Paris.

Schäfer, Henry, 2001: Triple Bottom Line Investing - Ethik, Rendite und Risiko in der Kapitalanlage, in: Zeitschrift für das gesamte Kreditwesen 54 (13), 740-744.

Sen, Amartya, 1999: Galbraith and the Art of Description, in: Sasson 1999, 139-146.

Shackle, George Lennox Sherman, 1972: Epistemics and Economics. A Critique of Economic Doctrines, Cambridge.

Solow, Robert, 1967: The New Industrial State or the Son of Affluence, in: Public Interest 9, 100-108.

Solow, Robert, 1997: How did Economics get that way, and what way did it get?, in: Daedalus 126 (1), Winter, 39-58.

Stigler, George/Becker, Gary, 1977: De Gustibus Non Est Disputandum, in: American Economic Review 67 (2), 76-90.

Summers, Lawrence, 1991: The Scientific Illusion in Empirical Economics, Scandinavian 
Journal of Economics 93 (2), 129-48.

Unseem, Michael, 1996: Investor Capitalism: How Money Managers Are Changing the Face of Corporate America, New York.

Vinten, Gerald, 2002: The corporate governance lessons of Enron, Corporate Governance, in: International Journal of Business in Society 2 (4), 4-9.

Waligorski, Conrad, 1990: The Political Theory of Conservative Economists, Lawrence (University of Kansas).

Webley, Simon/More, Elise, 2003, Does Business Ethics Pay? Ethics and financial performance,

London (Institute of Business Ethics).

Williamson, Oliver, 1970: Corporate Control and Business Behaviour, Englewood Cliffs/N. J.

Wood, Adrian, 1975: A Theory of Profits, Cambridge.

Korrespondenzanschrift:

Prof. Dr. Claus Dierksmeier

Associate Professor of Philosophy

Stonehill College/Philosophy Department

Easton, MA 02357, USA

E-Mail: clausdierksmeier@yahoo.de

Web: http://faculty.stonehill.edu/cdierksmeier/index.htm

\section{Betrachtungen des Luhmannschen Staatsverständnisses}

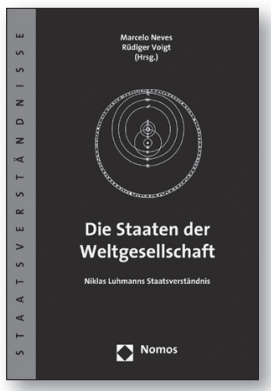

Die Staaten der Weltgesellschaft

Niklas Luhmanns Staatsverständnis

Herausgegeben von Prof. Dr. Marcelo Neves und Prof. Dr. Rüdiger Voigt, Universität der Bundeswehr München 2007, 242 S., brosch., 29,-€, ISBN 978-3-8329-2571-O (Staatsverständnisse, Bd. 13) 